\title{
The Dependence of Spatial Distribution of Excitement of Receptors on Their Stiles-Crawford Effect
}

\author{
Nachieketa K Sharma \\ Department of Physics, Institute of Technical Education \& Research, Siksha 'O' Anusandhan University, \\ Khandagiri Square, Bhubaneswar, OR, India
}

\begin{abstract}
The spatial distribution of excitement of receptors depends on the magnitude of amplitude of light integrated over the wavefront passing through the pupil as conditioned by the receptor's acceptance lobe. Because the peripheral zones of the pupil are less efficient in eliciting visual excitation than the pupil's centre, otherwise known as Stiles-Crawford effect of the first kind (SCE I), the spatial distribution of excitement of receptors will differ depending on their Stiles- Crawford effect. Assuming the funneling of light into the receptors through a single entering aperture the SCE I is integrated into the calculation of retinal diffraction images, in the manner of an apodization effect. And, in the absence of aberrations, the pupil apodization is chosen twice as narrow as suggested by the SCE I to compute modulation in the retinal light distributions. But using coherent illumination, a periodic target of rectangular profile and incorporating SCE I as pupil apodization we got a result where neither the intensity nor the modulation changes indicating absence of SCE I for a full pupil admitting a single coherent beam.
\end{abstract}

Keywords: Stiles-Crawford effect, Coherent illumination, Apodization, Rectangular grating.

\section{Introduction}

The obliquely incident light on the retina elicits a poor visibility response due to Stiles-Crawford effect of the first kind (SCE I) [1]. The change in the PSF produced by a gradual shading of the aperture towards its edge had been described as an apodization [2], because it reduces the height of the surrounding rings in the Airy disk. However, for the same oblique angle of incidence on the retina the SCE does not discriminate between a coherent and an incoherent beam [3]. But when an opportunity of interference is offered to the retina by way of adding another coherent beam to the existing one, retina's response is no more solely guided by the SCE I [4]. And so long a wavefront slope or an underlying phase is present in the light the retina can ill afford to ignore that. Hence a visual response in the manner of Stiles-Crawford (SCE I) diminution of effective brightness can be elicited by generating a wavefront slope at the retina [5]. When the wavefront slope at the retina is zero, the Poynting vector has also zero obliquity with no reduction in effective brightness. But with increasing wavefront slope the obliquity of the Poynting vector increases [6]. This is to say that the effective brightness is now controlled by the Poynting vectors and the SCE I brightness diminution is conditional on the obliquity of the Poynting vector at the retinal site where photon transduction takes place. The obliquity of the Poynting vectors somehow kills the interference effect in the retinal plane owing to rapid and irregular phase fluctuations in the incoherent illumination [6]. With the disappearance of the interference effect the SCE I brightness reduction for oblique incidence reappears [7]. This also excludes hitherto widely used and implemented effective shading of the wavefront amplitude before interference [8-13] as a possible explanation for SCE I. By treating the SCE I as a pupil apodization in the diffraction calculations of the retinal light distributions for a periodic rectangular target in coherent illumination I got a null result which backs the experimental findings, that unlike incoherent illumination, the SCE I as an apodization ceases to be effective in case of coherent illumination.

\section{Approach}

The change in the point spread function (PSF) produced by a gradual shading of the aperture toward its edge had been described as an apodization [2], because it reduces the height of the surrounding rings in the Airy disk. The reasonable assumption of light entering into a cone through a single transverse aperture allows us to incorporate the SCE I into the computation of retinal light distributions in the manner of an apodizing effect [14]. In the absence of aberrations this gives the identical prediction for effective retinal diffraction images with the photoreceptor waveguiding provided one is careful in multiplying the amplitude of the wavefront at each pupil location with the square root of the SCE I at that pupil location (square root because the SCE I has been measured for intensity and not amplitude) prior to the transformation leading to the retinal image light distribution [15]. Moreover unlike incoherent illumination, the non-linearity in intensity for coherent imaging does not allow the formulation of any general statements about the retinal image [16]. Thus the particular choice of test target and pupil opening (that too, in the absence of aberrations and defocus) will have its own kind of imaging characteristics in coherent illumination. What is then the series of transformations in case of coherent 
imaging ultimately leading to the fundamental imaging property of a human eye which is contained either in the modulation transfer function in the Fourier domain or in the image intensity distribution of a point object in the space domain? The amplitude of light in the pupil plane is the Fourier transform of the amplitude in the plane of the rectangular grating. This distribution represents the spatial frequency content of the amplitude displayed at distances from the geometrical image of the source proportional to the spatial frequencies.

Ultimately the amplitude distribution of light in the plane of the retina is subjected to a non-linear transformation, that is, squaring, and the resulting intensity distribution constitutes the retinal image [16].

This I have done by evaluating the modulation, an effective parameter for image quality assessment by choosing a rectangular wave grating as a test target. When the target, here the periodic rectangular wave grating is illuminated by spatially coherent light, the amplitude of light coming out of the target plane is displayed as a diffraction image in the plane of the pupil. It is then filtered, here the SCE I acting as a neutral density wedgeshaped filter. After this it is resynthesized as a distribution of amplitude of light in the retinal plane. Finally the amplitude is squared at each point in the image giving rise to image intensity distribution leading to modulation.

The approach is to incorporate SCE I in retinal diffraction image computation as an apodization in coherent illumination and to get results which confirm apodization's ineffectiveness. First, the Fourier transform of the amplitude transmittance of a vertical rectangular wave grating with average amplitude $a$, modulation $b$, period $\mathrm{p}$ and duty cycle $\alpha$ is taken [17]

to obtain the object spatial frequency spectrum $a(u, v)$

$$
A(x, y)=(a-b+2 \alpha b)+\frac{4 b}{\pi} \sum_{n=1}^{\infty} \frac{\sin (n \alpha \pi)}{n} \cos (n \omega x)
$$

$$
\begin{aligned}
\text { as } \iint_{-\infty}^{\infty}\left[(a-b+2 \alpha b)+\frac{4 b}{\pi} \sum_{n=1}^{\infty} \frac{\sin (n \alpha \pi)}{n} \cos (n \omega x)\right] e^{-i(u x+v y)} d x d y \\
\quad=(a-b+2 \alpha b) \delta(u, v)+\frac{2 b}{\pi} \sum_{n}^{\infty}[\delta(u-n \omega, v)+\delta(u+n \omega, v)]
\end{aligned}
$$

by utilizing the properties of Dirac delta function.

Multiplying this object Fourier spectrum with the amplitude transfer function $\mathrm{e}^{\frac{-\left(\mathrm{u}^{2}+\mathrm{v}^{2}\right)}{\sigma^{2}}}=$ $\mathrm{e}^{-0.105\left(\mathrm{u}^{2}+\mathrm{v}^{2}\right)}$, (where $\sigma=3.086$ ) [18] which is mathematically identical to the pupil function within a coordinate scaling operation for spatially coherent illumination, I got the image spatial frequency spectrum as

$$
a^{\prime}(u, v)=e^{-0.105\left(u^{2}+v^{2}\right)}\left[(a-b+2 \alpha b) \delta(u, v)+\frac{2 b}{\pi} \sum_{n}^{\infty}\{\delta(u-n \omega, v)+\delta(u+n \omega, v)\}\right]
$$

Inverse transforming this spectrum and again utilizing the properties of Dirac delta function it is found that the output field is: $\mathrm{A}^{\prime}(\mathrm{x}, \mathrm{y})=(\mathrm{a}-\mathrm{b}+2 \alpha \mathrm{b})+\frac{4 \mathrm{~b}}{\pi} \sum_{\mathrm{n}=1}^{\mathrm{n}^{\prime}} \mathrm{e}^{-0.105\left(\mathrm{u}^{2}+\mathrm{v}^{2}\right)} \quad \cos (\mathrm{n} \omega \mathrm{x})$

As the amplitude function $\mathrm{e}^{-0.105\left(\mathrm{u}^{2}+\mathrm{v}^{2}\right)}$ by definition, is zero outside the pupil and 1 inside, we can write the upper limit $n^{\prime}$ of $n$ is such that $n \omega \leq 1$. Finally, the intensity distribution in the image plane is given by the squared modulus of this output field.

$$
\mathrm{B}^{\prime}(\mathrm{x}, \mathrm{y})=\left[\begin{array}{ll}
(\mathrm{a}-\mathrm{b}+2 \alpha \mathrm{b})+\frac{4 \mathrm{~b}}{\pi} \sum_{\mathrm{n}=1}^{\mathrm{n}^{\prime}} \mathrm{e}^{-0.105\left(\mathrm{u}^{2}+\mathrm{v}^{2}\right)} & \cos (\mathrm{n} \omega \mathrm{x})
\end{array}\right]^{2}
$$

The modulation $\left(\mathrm{M}=\frac{\mathrm{B}_{\max }^{\prime}-\mathrm{B}_{\min }^{\prime}}{\mathrm{B}_{\max }^{\prime}+\mathrm{B}_{\min }^{\prime}}\right)$ is found out by evaluating the maximum and minimum intensity at $\omega x=0$ and $\omega \mathrm{x}=\pi$ respectively.

I have compared the intensity without and with SCE I as pupil apodization for different duty cycle of the grating. The modulation M is also compared in the absence and presence of SCE I, again for various duty cycle.

\section{Results And Discussion}

It is to be noted that throughout this paper the spatial frequency is normalized with respect to the cut-off spatial frequency. Thus, for example, when $\omega=0.2$ is mentioned, it means the spatial frequency discussed is just $20 \%$ of the cut-off spatial frequency for coherent illumination. Similar is the case with reduced distance $(\omega x)$. In order to understand the response of modulation to spatial coherence of the entering beams in presence of the Stiles Crawford effect of the first kind, two categories of relations are studied, first, the variation of intensity with reduced distance, and next, the response of modulation to different normalized spatial frequencies. This is done both by modeling Stiles Crawford effect of the first kind as a pupil apodization and without it. To give richness to the study, different duty cycles are taken into consideration. Again, two types of normalized spatial frequencies, low and high are chosen. 


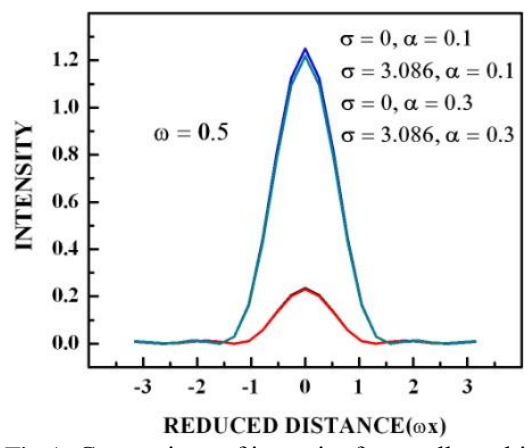

Fig. 1: Comparison of intensity for small $\omega$ taking $\alpha=0.1 \& 0.3$.

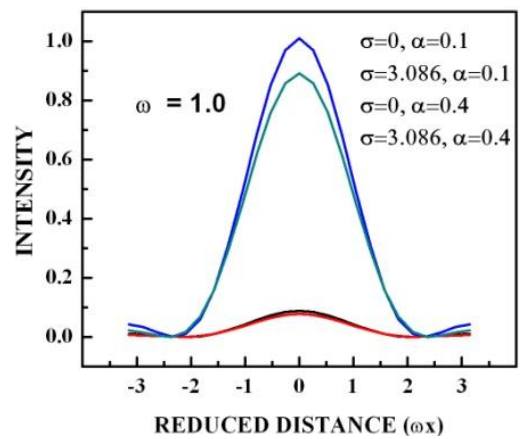

Fig.3: Comparison of intensity for large $\omega$ taking $\alpha=0.1 \& 0.4$

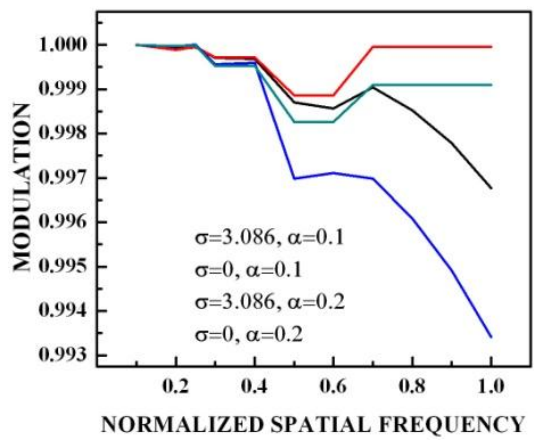

Fig.5: Comparison of modulation for $\alpha=0.1 \& 0.2$.

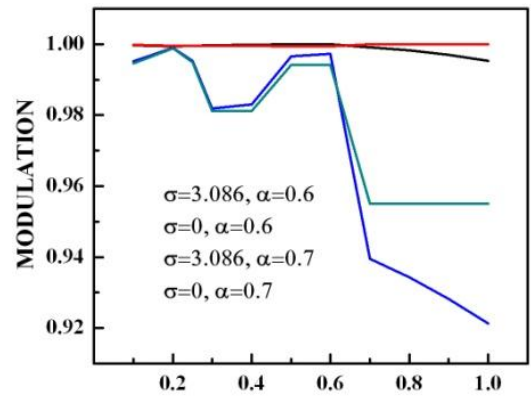

NORMALIZED SPATIAL FREQUENCY

Fig.7: Comparison of modulation for $\alpha=0.6 \& 0.7$.

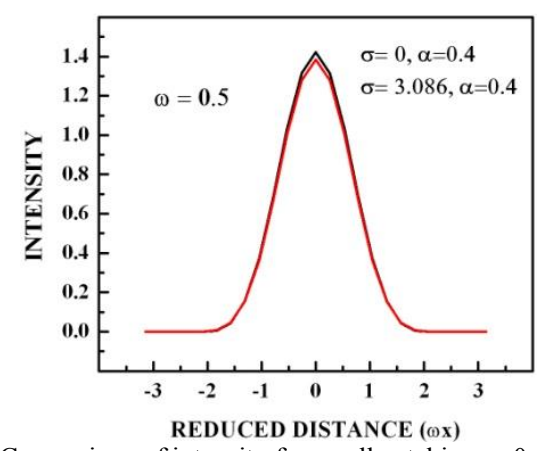

Fig.2: Comparison of intensity for small $\omega$ taking $\alpha=0.4$

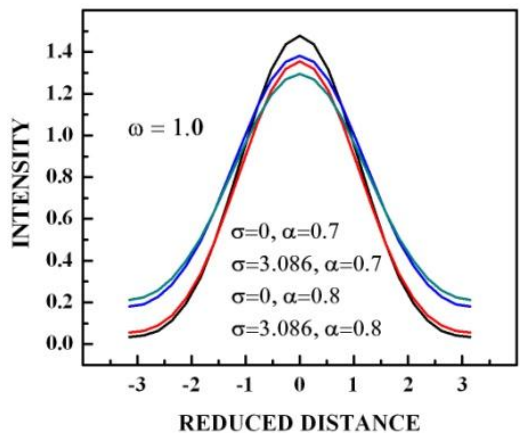

Fig.4: Comparison of intensity for large $\omega$ taking $\alpha=0.7 \& 0.8$

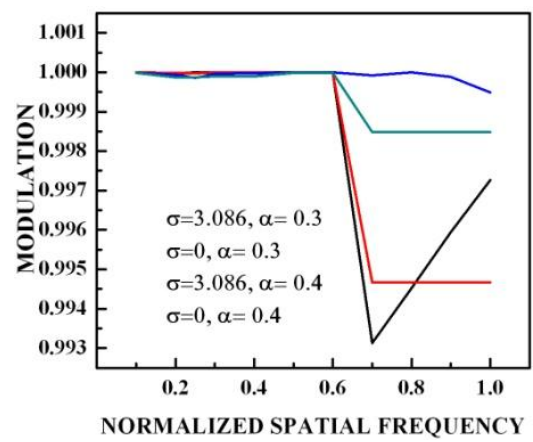

Fig.6: Comparison of modulation for $\alpha=0.3 \& 0.4$.

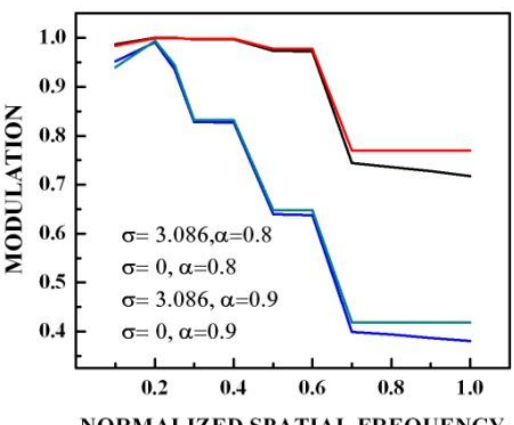

Fig.8: Comparison of modulation for $\alpha=0.8 \& 0.9$ 
The intensity distribution in the images of periodic rectangular wave targets, formed by a human eye apodized with SCE-I under coherent illumination was computed (from figs $1-4$ ), both for a human eye apodized with the SCE I and in the absence of it for different values of the duty cycle of the periodic target chosen. It is seen from the figures that for coherent illumination, apodization treatment does not modify the intensity in each case of the duty cycle for both low and high normalized spatial frequency.

Similarly, the intensity expression is used to find out the modulations in the images plotted graphically from figs. 5-8. From the graphs it is evident that the modulation changes only by $4 \%$ throughout in almost all cases of the duty cycles taken here indicating almost absence of directionality for a SCE-I apodized human eye under coherent illumination over the entire spatial frequency range for a rectangular wave target.

\section{Conclusion}

The result thus arrived at taking into account a rectangular wave periodic grating strengthens the experimental finding [4-5] of the absence of the traditional SCE-I directionality for the entire human pupil under coherent illumination. And it raises the fundamental question of how light beams capable of interference, finally affect retinal image formation differing distinctly from beams not able to interfere.

\section{References}

[1]. W. S. Stiles and B. H. Crawford, “The Luminous Efficiency of Rays Entering the Pupil at Different Points,” Proc. Roy. Soc. B, 112, 428-450 (1933)

[2]. P. Jacquinot, P. Bougron, and B. Dossier, "Calcul et realisation des distributions d'amplitude pupillaire, permettant a suppression des franges laterale dans les figures de diffraction," in La Theorie des Images Optiques, P. Fleury, A. Marechal, and C. Anglade, eds. (Editions de la Revue d'Optique, 1949), pp. 183-193.

[3]. L. J. Bour and J. C. M. Verhoosel, "Directional Sensitivity of Photoreceptors for Different Degrees of Coherence and Direction of Polarization of the Incident Light," Vis. Res. 19, 717-719 (1979)

[4]. B. Vohnsen and D. Rativa, "Absence of an Integrated Stiles-Crawford Function for Coherent Light," Journal of Vision, 19, 1-10 (2011)

[5]. S. Castillo, and B. Vohnsen, "Exploring the SCE-I with Coherent Light and Dual Maxwellian Sources," Appl. Optics 52, A1-A8 (2013).

[6]. G. Westheimer, "Retinal Light Distributions, the Stiles-Crawford Effect and Apodization," J. Opt. Soc. Am. A 30, 1417-1421 (2013)

[7]. B. Vohnsen, I. Iglesias, and P. Artal, “Guided Light and Diffraction Model of Human-Eye Photoreceptors,” J. Opt. Soc. Am. A 22, 2318-2328 (2005)

[8]. H. Metcalf, "Stiles-Crawford Apodization,” J. Opt. Soc. Am. 55, 72-74.1411-1416 (1965).

[9]. James P. Carroll, “Apodization Model of the Stiles-Crawford Effect,” J. Opt. Soc. Am. A 70, 1155-1156 (1980)

[10]. D. A. Palmer, "Stiles-Crawford Apodization and the Campbell Effect," J. Opt. Soc. Am. A 2, 1371-1374 (1985).

[11]. Pablo Artal, "Incorporation of Directional Effects of the Retina into Computations of Optical Transfer Functions of Human Eyes," J. Opt. Soc. Am. A 6, 1941-1944 (1989)

[12]. X. Zhang, M. Ye, A. Bradley, and L. Thibos, "Apodization by the Stiles-Crawford Effect Moderates the Visual Impact of Retinal Image Defocus," J. Opt. Soc. Am. A 16, 812-820 (1999).

[13]. D. A. Atchison, D. H. Scott, N. C. Strang, and P. Artal, "Influence of Stiles-Crawford Apodization on Visual Acuity, "J. Opt. Soc. Am. A 19, 1073-1083 (2002).

[14]. G. Westheimer, "Specifying and Controlling the Optical Image on the Retina, Prog. Retinal Eye Res. 25, 19-42 (2006)

[15]. B. Vohnsen, "Photoreceptor Waveguides and Effective Retinal Image Quality," J. Opt. Soc. Am. A 24, 597-607 (2007)

[16]. G. Westheimer, “The Maxwellian View,” Vis. Res. 6, 669-682 (1966)

[17]. P. K. Katti, K. Singh, and A. K. Kavathekar, "Effect of an Apodizing Screen on the Rectangular and Triangular Wave Response of a Circular Aperture with Incoherent Incident Light," Appl. Optics 9, 129-134 (1970).

[18]. R.A.Applegate \& V. Lakshminarayanan, "Parametric Representation of Stiles-Crawford Functions: Normal Variation of Peak Location and Directionality," J. Opt. Soc. Am. A 10, 1611-1623 (1993). 